\title{
Wavelength-dependent optical force on elliptical silver cylinders at plasmon resonance
}

\author{
Carsten Rockstuhl and Hans Peter Herzig \\ University of Neuchâtel, Institute of Microtechnology, Rue A.-L. Breguet 2, CH-200o Neuchâtel, Switzerland \\ Received May 11, 2004
}

\begin{abstract}
We use rigorous diffraction theory to analyze the force on elliptical cylinders made from silver as a function of the elongation. We find that, when the wires are illuminated at the plasmon wavelength and placed in a highly focused Gaussian beam, they are attracted toward the optical axis if the waist of the laser is behind the wire and repelled if the waist is before the wire. Also, the force distributions of illumination wavelengths smaller and larger than the plasmon wavelength are analyzed. (C) 2004 Optical Society of America

OCIS codes: $240.6680,290.0290,350.4990,350.3950$.
\end{abstract}

Illuminating silver or gold nanoparticles at appropriate wavelengths excites a surface plasmon, which is a resonant oscillation of the free electrons in the material. ${ }^{1} \quad$ For particles with a noncircular or nonspherical geometry, rigorous diffraction theory must be used to determine precisely the wavelength for which a plasmon is excited. At the resonance a large scattering cross section ${ }^{2}$ (SCS) and an enhanced near-field amplitude are observed. The enhanced near field in the vicinity of the particles could be applied, for example, to a scanning near-field optical microscope, in which a trapped metallic particle serves as a highly spatially confined probe. ${ }^{3}$ Another application is the photonic force microscope. ${ }^{4}$ For both applications an optical tweezer is used to trap the particles. ${ }^{5}$ The optical tweezer is a highly focused laser beam that exerts a force on the particle, such that it can be stably trapped at a certain point. This is possible if all the forces acting on the particle are in equilibrium. To describe the working principle of an optical tweezer applied to metallic particles two main approaches have been used in the past. The first is a ray-optical model that can be used if the particle is significantly larger than the wavelength. ${ }^{6}$ If the particle is much smaller than the wavelength, a dipole model is used. ${ }^{7}$ For spherical particles between these size domains, Mie theory can be used to calculate the forces on metallic particles precisely. ${ }^{8}$

In this Letter we use rigorous diffraction theory to determine the force on metallic wires. Recently, Arias-Gonzáles and Nieto-Vesperinas ${ }^{7}$ calculated rigorously the force on two-dimensional circular cylinders under the plasmon resonance condition by means of the extinction theorem boundary condition, mainly for plane-wave or weakly focused Gaussian-beam illumination above substrates. We analyze the force on silver elliptical cylinders illuminated by a highly focused laser beam as a function of the axis ratio and the wavelength by using the multiple multipole method. $^{9}$ We restrict ourselves to two-dimensional structures. For excitation of a plasmon the electric field vector must have a component that oscillates perpendicularly to the geometrical cross section; thus a TM-polarized field is used for illumination. The numerical procedure for calculating the force consists of two steps. First the electric and magnetic fields are calculated along the surface of the cylinder. The wires are illuminated with a highly focused Gaussian beam whose waist equals one wavelength $(\omega=\lambda)$; the amplitude of the illuminating Gaussian beam is $6.9 \mathrm{~V} / \mathrm{m}$, which is the amplitude of a three-dimensional beam with a power of $100 \mathrm{~mW}$. The dielectric constants for silver were taken from Ref. 10, and the area of the elliptical cylinders was kept constant at $A=\pi(20 \mathrm{~nm})^{2}$. The different elongations were obtained by multiplying or dividing the radii by use of factors $\sqrt{2}, \sqrt{3}$, and $\sqrt{4}$. If the radius in the longitudinal direction $r_{1}$ was multiplied by the given factor, the opposite transverse radius $r_{2}$ was divided by the same factor to maintain the area.

In the second step the force exerted on the cylinder is calculated by applying the Maxwell stress tensor. Only the time average of the electromagnetic force is observed at optical frequencies. For a wire embedded in an isotropic medium the force is calculated with ${ }^{11}$

$$
\begin{aligned}
\langle\mathbf{F}\rangle= & \int_{S}\left\{\frac{\epsilon}{2} \operatorname{Re}\left[(\mathbf{E} \cdot \mathbf{n}) \mathbf{E}^{\star}\right]-\frac{\epsilon}{4}\left(\mathbf{E} \cdot \mathbf{E}^{\star}\right) \mathbf{n}\right. \\
& \left.+\frac{\mu}{2} \operatorname{Re}\left[(\mathbf{H} \cdot \mathbf{n}) \mathbf{H}^{\star}\right]-\frac{\mu}{4}\left(\mathbf{H} \cdot \mathbf{H}^{\star}\right) \mathbf{n}\right\} \mathrm{d} l^{\prime},
\end{aligned}
$$

where $S$ is the circumference of the wire and $\mathbf{n}$ is the normal to the surface.

The normalized SCSs for wavelengths from 300 to $450 \mathrm{~nm}$ assuming plane-wave illumination are shown in Fig. 1. The ovals show the cylinder orientation with respect to the propagation direction of the illuminating beam for the two outermost elongated cylinders. The arrangement for all the other cylinders is the same. An excitation of a plasmon appears as a peak in the SCS. $\quad r_{1}$ is the radius of the wire parallel to the optical axis, and $r_{2}$ is the perpendicular radius. It can be seen that the plasmon wavelength can be tuned over approximately $100 \mathrm{~nm}$ and depends solely on the axis ratio $\left(R=r_{2} / r_{1}\right)$ of a given material. The larger the axis ratio, the larger the redshift as well as the FWHM of the excited plasmon. The reason for an increased FWHM for the wires with a larger plasmon 


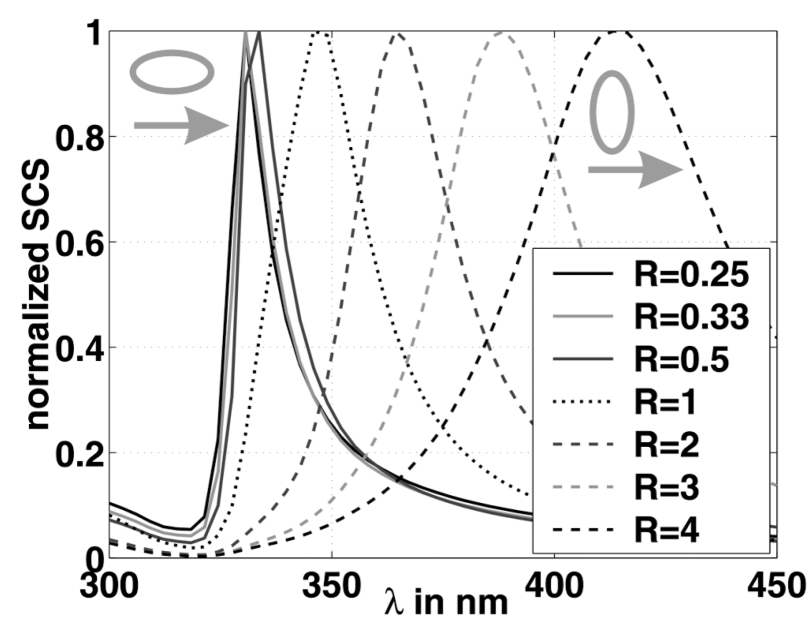

Fig. 1. Normalized SCS of silver elliptical cylinders as a function of elongation.

wavelength is attributed mainly to phase retardation inside the wire and to an increasing imaginary part in the dielectric constant. Both effects cause damping and broadening of the response and overall smoothing of the SCS. The strength of the SCS is lowest for the wire with the lowest aspect ratio. The excited plasmons are dominated by a dipole excitation. An alternative way to control the plasmon wavelength is to change the cylinder size. Increasing the size of the cylinders will redshift the wavelength, and the overall achievable shift can be comparable with the difference of the plasmon wavelength between two subsequent chosen elongations. However, the overall achievable wavelength range is smaller, and the major drawback is an increase of the width of the plasmon peak. We restrict our discussion hence to the possibility of changing the plasmon wavelength by changing just the elongation.

We calculated the force for different elongations as a function of the wavelength. A typical force distribution at the resonance wavelength is shown in Fig. 2(a) for the circular cylinder $\left(r_{1}=r_{2}=20 \mathrm{~nm}\right)$. The illumination wavelength was $\lambda_{\text {Resonance }}=340 \mathrm{~nm}$. The distribution of the force at a nonresonant wavelength is shown in Figs. 2(b) $(\lambda=450 \mathrm{~nm})$ and 2(c) $(\lambda=1500 \mathrm{~nm})$. The amplitude distribution of the illuminating beam, which propagates in the positive $z$ direction, is shown in the background, and the forces are shown on a nonlinear scale to emphasize the direction. In the case of a wavelength close to the plasmon wavelength [Figs. 2(a) and 2(b)] the longitudinal force is always positive independent of the wire position, meaning that the wires are accelerated along the optical axis. For a wavelength far from the plasmon resonance [Fig. 2(c)], the gradient force dominates the scattering force and the longitudinal force takes negative values on the optical axis behind the waist. The object could be trapped at a point a short distance behind the waist. The transverse force in the resonant case attracts the wire toward the optical axis if it is positioned before the waist and repels it from the optical axis if the wire is situated behind the waist. For a larger wavelength the wire is attracted toward the optical axis over an increased range, including behind the waist. For a shorter wavelength the wire is repelled even at positions significantly before the waist. Such behavior can be explained by calculating the polarizability $\alpha$ of an elliptical cylinder, assuming a dipole model. For an elliptical cylinder illuminated with a TM-polarized wave, $\alpha$ is proportional to $\left(\epsilon_{i}-\epsilon_{a}\right)(1+R)\left(R \epsilon_{a}+\epsilon_{i}\right)^{-1}$, where $\epsilon_{i}$ is the dielectric constant of the cylinder and $\epsilon_{a}$ is the dielectric constant of the surrounding medium. The real part of $\epsilon$ for silver in the relevant wavelength region is negative, and for higher wavelengths this value decreases. For a simplified rough analysis we assume first that the imaginary part for the corresponding wavelength is small and hence can be neglected and discuss subsequently its influence on the behavior.

One can see that a plasmon is excited if the denominator becomes zero, which explains the increase of the plasmon wavelength for higher aspect ratios. For wavelengths smaller than the plasmon wavelength, $\alpha$ becomes negative because the numerator, given mainly by $\left(\epsilon_{i}-\epsilon_{a}\right)$, is always smaller than zero and $R \epsilon_{a}+\epsilon_{i}$ is positive. The gradient force, $F_{\text {grad }}$, proportional to $\alpha \nabla\left(|E|^{2}\right)$, points in the opposite direction to the gradient. This means that in this wavelength regime the wires are basically repelled from the optical axis. For wavelengths higher than the plasmon wavelength, the denominator also becomes negative and $\alpha$ becomes positive, which will cause an attraction of the wire toward the optical axis.

The entire wavelength dependence of the force for a wire whose minor axis is $r_{1}=20 \mathrm{~nm} / \sqrt{3}$ and whose major axis is $r_{2}=20 \mathrm{~nm} \sqrt{3}$ can be seen in Fig. 3(a),
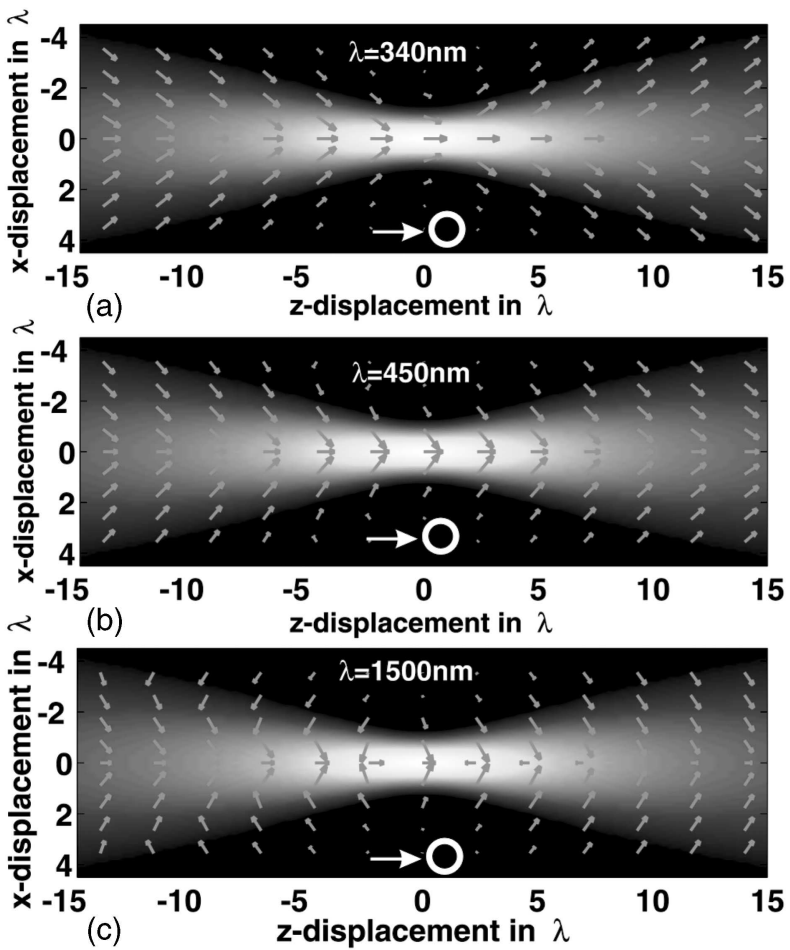

Fig. 2. Force on a silver circular cylinder $\left(r_{1}=r_{2}=20 \mathrm{~nm}\right)$ illuminated with a Gaussian beam at (a) the plasmon resonance wavelength $(\lambda=340 \mathrm{~nm})$, (b) an off-resonance wavelength $(\lambda=450 \mathrm{~nm})$, and (c) a wavelength far from resonance $(\lambda=1500 \mathrm{~nm})$. 


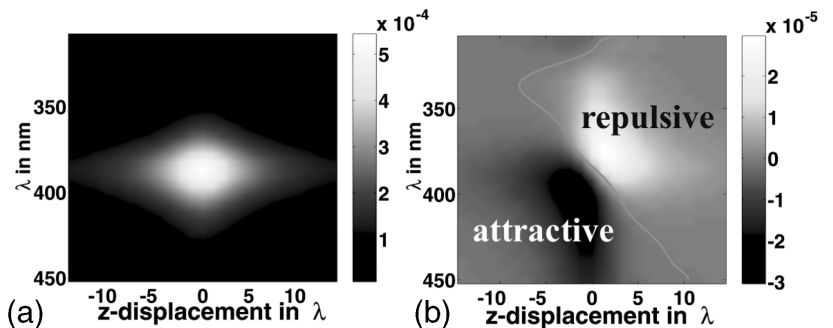

Fig. 3. (a) On-axis longitudinal force and (b) transverse force evaluated parallel to the axis at $x=0.44 \lambda$ for an elliptical cylinder with $r_{1}=20 \mathrm{~nm} / \sqrt{3}$ and $r_{2}=20 \mathrm{~nm} \sqrt{3}$ as a function of wavelength.
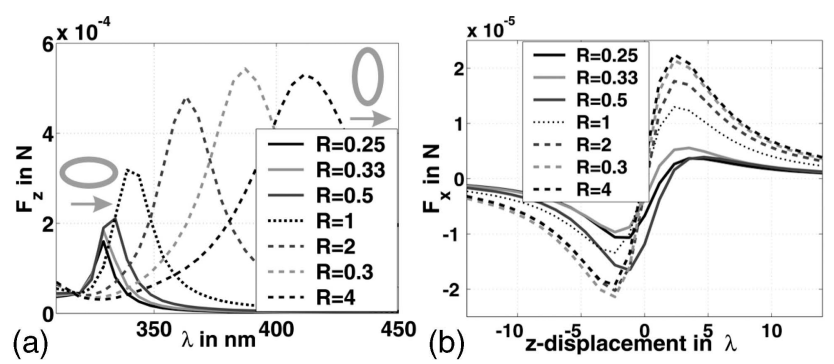

Fig. 4. (a) Longitudinal force in the center of the waist as a function of wavelength and (b) transverse force along the optical axis at $x=0.44 \lambda$ for the resonance wavelength.

where the on-axis longitudinal force as a function of the wavelength is shown. The longitudinal force is always maximum at the plasmon wavelength. This component is dominated by the scattering force, which is proportional to the intensity and the square of the absolute value of $\alpha$, which is highest at the resonance. Correspondingly, trapping of these objects at the plasmon wavelength by use of a Gaussian beam is not possible, as the gradient force cannot equal the scattering force, which is the necessary condition for trapping. Trapping is possible instead by use of a wavelength that is significantly larger than the plasmon wavelength, as shown in Fig. 2(c). In Fig. 3(b) the transverse force evaluated off-axis along the $z$ axis at $x=$ $0.44 \lambda$ is shown. A positive force $F_{x}$ indicates a repulsion from the axis; a negative force, an attraction. It is important to note that the same distribution was qualitatively found for each of the wires. The transverse force acting on the wire is strongest slightly behind the waist at a wavelength shorter than the resonance wavelength and smallest at a point slightly before the waist, at a wavelength larger than the resonance. If the imaginary part of the dielectric constant were to be zero and if the application of the dipole approximation did not introduce a small error, the change of the sign for the force would appear at exactly the resonance wavelength, independent of the $z$ displacement of the wire. The gradient force at this point is zero. ${ }^{7}$ The nonnegligible imaginary part as well as the finite size of the wire will cause a change of sign at different $z$ displacements for different wavelengths.

For a comparison of the differently elongated wires, in Fig. 4(a) we show the longitudinal force acting on the wires in the center of the waist as a function of the wavelength. The transverse force at the resonance wavelength evaluated parallel to the optical axis at $x=0.44 \lambda$ is shown in Fig. 4(b). The longitudinal force becomes maximum at the plasmon wavelength. The behavior at the plasmon resonance wavelength of the transverse force is comparable for all the wires, independent of the elongation direction. The wires are repelled at positions approximately after the waist and attracted for positions before the waist.

In summary, we have analyzed the force on elliptical silver cylinders as a function of the cylinder elongation and of wavelength. The longitudinal force is strongest at the plasmon wavelength. The transverse force undergoes a change of sign at the plasmon wavelength. Because of the nonnegligible size of the wire as well as the imaginary part of the dielectric constant, this change of sign appears at different $z$ positions as a function of the wavelength. For wavelengths shorter than the plasmon wavelength, the wires are repelled from the optical axis. For a larger wavelength, the wires are attracted. Such a different response to an applied focused wave field offers a fascinating application using focused beams for sorting of metallic particles in accordance with their shape, orientation, or perhaps material composition by means of cascaded beams, where subsequent levels work at different wavelengths. Although it is only analyzed for a two-dimensional structure, the basic physical response will be the same for three dimensions, such that this capability would be useful, e.g., for selecting nanoparticles with a well-defined resonance wavelength for plasmon-based waveguides or for the use of particles applied to surface-enhanced Raman spectroscopy. For a complete description of the flow of objects in wave fields, torque must additionally be taken into account, as it will cause an alignment of the object with respect to the optical axis.

This research was supported by the European Union under grant IST-2000-26479. C. Rockstuhl's e-mail address is carsten.rockstuhl@unine.ch.

\section{References}

1. J. P. Kottmann and O. J. F. Martin, Appl. Phys. B 73, 299 (2001).

2. M. Born and E. Wolf, Principles of Optics (Cambridge U. Press, Cambridge, England, 1999).

3. T. Sugiura, T. Okada, Y. Inouye, O. Nakamura, and S. Kawata, Opt. Lett. 15, 1663 (1997).

4. A. Rohrbach, E. L. Florin, and E. H. K. Stelzer, Proc. SPIE 4431, 75 (2001).

5. A. Ashkin, Phys. Rev. Lett. 24, 156 (1970).

6. P. C. Ke and M. Gu, Appl. Opt. 38, 160 (1999).

7. J. R. Arias-Gonzáles and M. Nieto-Vesperinas, J. Opt. Soc. Am. A 20, 1201 (2003).

8. H. Furukawa and I. Yamaguchi, Opt. Lett. 23, 216 (1998).

9. C. Hafner, Post-Modern Electromagnetics (Wiley, New York, 1999).

10. P. B. Johnson and R. W. Christy, Phys. Rev. B 6, 4370 (1972).

11. M. Lester and M. Nieto-Vesperinas, Opt. Lett. 24, 936 (1999). 